\title{
Multifunctional Carbon Fibre Tapes for Automotive Composites
}

\author{
V. Koncherry ${ }^{1} \cdot$ P. Potluri $^{1} \cdot$ A. Fernando ${ }^{1}$
}

Received: 29 September 2016 / Accepted: 24 October 2016/Published online: 18 November 2016

(C) The Author(s) 2016. This article is published with open access at Springerlink.com

\begin{abstract}
Cabon fibre composites are used where mechanical performance such as strength, stiffness and impact properties at low density is a critical parameter for engineering applications. Carbon fibre flat tape is one material which is traditionally used to manufacture threedimensional composites in this area. Modifying the carbon fibre tape to incorporate other functions such as stealth, electromagnetic interference, shielding, de-icing, self-repair, energy storage, allows us to create multi-functional carbon fibre tape. Researchers have been developing such material and the technology for their manufacture in order to produce multifunctional carbon fibre based components more economically and efficiently. This paper presents the manufacturing process of a metallised carbon fibre material for a chopped fibre preforming process that uses electromagnets for preforming instead of traditional suction airflow fibre deposition. In addition, the paper further presents mechanical and magneto-static modelling that is carried out to investigate the bending properties of the material produced and its suitability for creating 3D preforms.
\end{abstract}

Keywords Tape $\cdot$ Magnetic properties $\cdot$ Finite element analysis $\cdot$ Carbon fibre $\cdot$ Preform

\section{Introduction}

Original equipment manufacturers of the automotive industry are facing strict government directives to reduce fuel consumption, thus reducing the emissions to meet the commitments of the Kyoto protocol [1-3]. A proven method of meeting the emission requirements is by reducing the weight of the automobiles [4, 5]. As published, reduction in weight of an automobile by $20 \%$ reduces the fuel consumption by $8.4 \%$ [6]. To accomplish the weight reduction in automobiles, it is possible to reduce the weight of the moulded components used in automobiles by $50-70 \%$ by replacing metallic materials with composites [7-9]. However,

\footnotetext{
A. Fernando

anura.fernando-2@manchester.ac.uk
}

1 Robotics \& Textile Composites Group, North West Composites Centre, School of Materials, The University of Manchester, Manchester M13 9PL, UK 
the manufacturing cost of composites based automobile moulded components remains one of the main concerns of automobile manufacturers since pure carbon fibre is 15 times more expensive than steel $[10,11]$. To reduce the cost of the composites requires low cost carbon fibres as well as high throughput production processes [12].

Past research shows that chopped fibre preforming processes, such as P4 (Powder Programmable Preforming Process) and DFP, allow fibre deposition to be carried out approximately at a speed of $4 \mathrm{~kg} / \mathrm{min}$ (Glass Fibre), on to the mould surface as seen in Fig. 1 [13-21]. The moulding process that uses chopped carbon fibres and the binder resin is called the Directed Carbon Fibre Preforming (DCFP). This is an automated process through which chopped carbon fibres can be placed on to a $3 \mathrm{D}$ surface [22-24] through air evacuation. The process consists of chopping fibres and blowing them on to a mould surface to create the necessary moulded components. The binder which helps to solidify this shape is introduced during the placement of the fibres. However as reported by Harper et al. [25], the low density carbon fibres tend to get disrupted, particularly on vertical surfaces of the mould tool, due to the suction air streams.

To overcome this drawback in the DCFP process, the composite research team at the University of Manchester has developed a metallised carbon fibre tow that can be

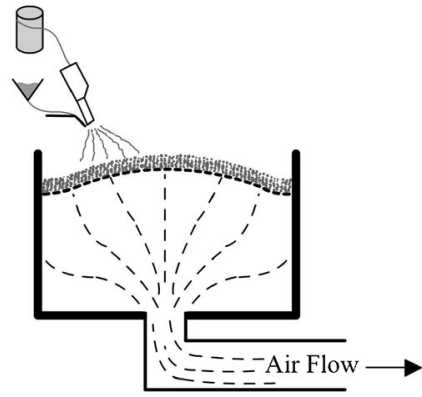

(a)

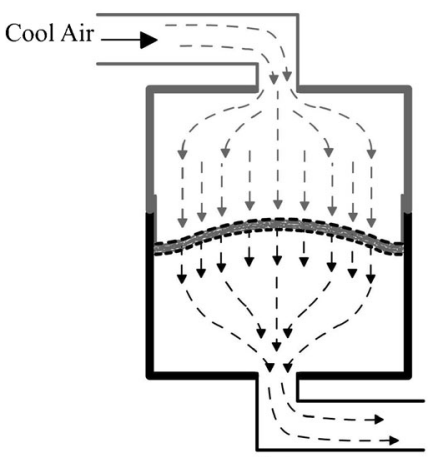

(c)

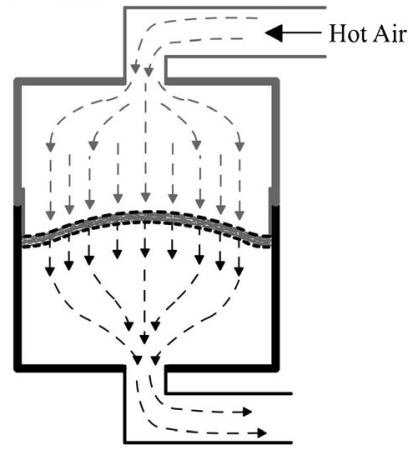

(b)

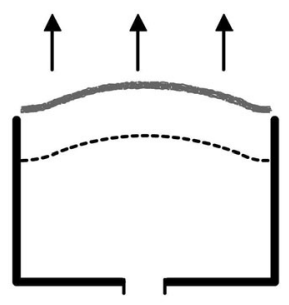

(d)

Fig. 1 The conventional DFP process [13]; a Fibre deposition, chopped fibre and binder applied to lower screen, held by vacuum (b) Consolidation, upper screen compacts preform, hot air cures binder (c) Cooling, ambient air cools preform (d) demoulding, preform removed from the lower screen [13] 
placed on mould surfaces using a magnetic field. This material is aimed to be used in a mould that has an electromagnetic field as illustrated in Fig. 2 for tow placement instead of suction air, as in the case of the DCFP process, thereby avoiding the carbon fibre disruption. The concept of depositing metallised carbon tow on to a mould surface, in the presence of a magnetic field, with the help of DCFP has the same economies as that of the DCFP process due to the similarity in the tooling and accessories used in the preforming process [26]. In addition, the ability to handle finished composite components manufactured using multifunctional Carbon Fibres (MCF) by electromagnetic pick and place robots, provides benefits in the subsequent assembly stages as well. All these advantages of using MCF, together with the reduced manufacturing times compared to other preforming processes like tow placement or weaving, makes MCF based Directed Carbon Fibre Preforming one of the main contenders in the composites manufacturing industries.

To visualise the magnetic energy for MCF tow placement, in an electromagnetic field similar to that encountered in the preforming process, it is important to investigate its magnetostatic properties. Further, in order to observe the material's capacity to conform to the curvatures of the mould surfaces, it is important to carry out experimental and theoretical studies. These experiments and the associated finite element based analysis are presented in this paper.

\section{Process Development}

In order to place carbon fibre tow on to a mould surface using a magnetic field, the tow needs partial metallisation. Here, an optimum metallisation needs to be carried out to ensure that the weight advantage carbon fibres have is not negated by the metallisation. To achieve these criteria, the MCF was designed with transverse lines of ferromagnetic metal powder placed at optimum predetermined distances, which was determined through extensive, trial and error, experimental work.

Fig. 2 Chopped fibre applied to mould and held by electromagnetic force

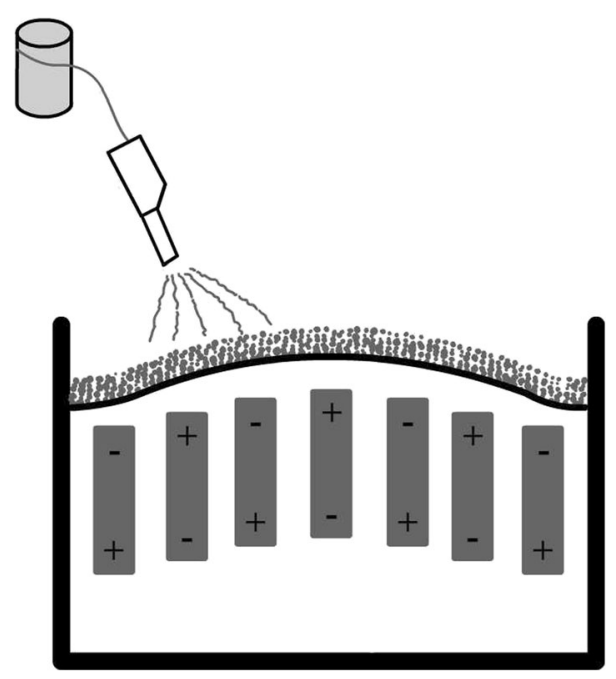




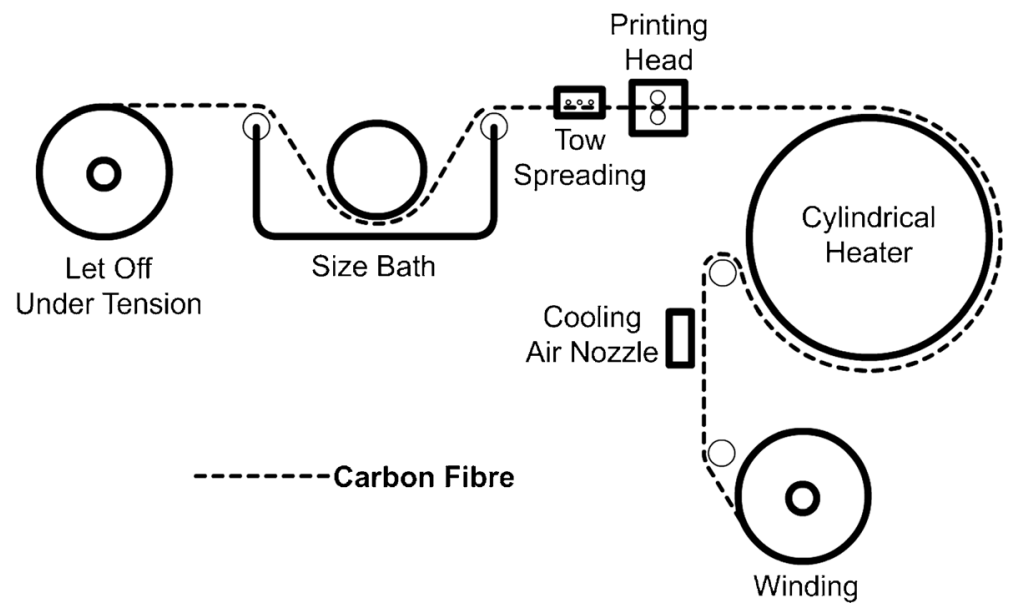

Fig. 3 Schematic diagram of the process for printing of metallic grating

In developing a manufacturing process to create MCF tow with the above features, a prototype carbon tow-printing machine was developed. The process sequence of this machine is shown in Fig. 3. The specific properties of the MCF tow this produces, depends on the subsequent applications for which it will be used.

The prototype machine built to manufacture the MCF tow uses water during the manufacturing process to prevent fibre damage. A paste made of nickel powder as seen in Fig. 4 mixed with Hexion EPI RZI water based epoxy was used as the metallic printing compound. Experiments conducted have confirmed that, printing the ferromagnetic paste provides one of the best methods of carbon tow metallisation, ensuring firm adhesion of the metal powder on to the tow as depicted in Fig. 5.

The visual appearance of the MCF in a real product can be seen from the example below, where an automobile spare-wheel-well was constructed using the multifunctional carbon fibres as shown in Fig. 6.

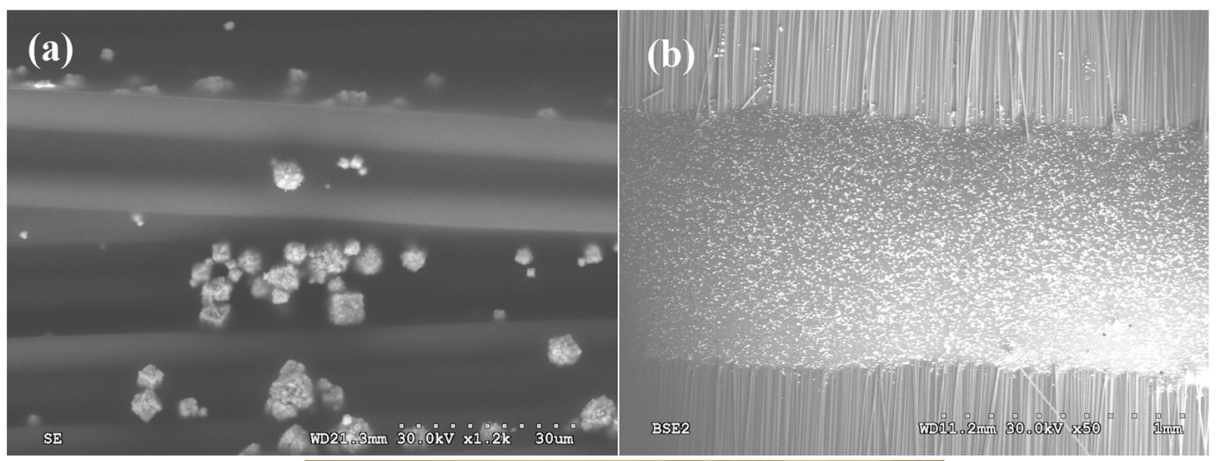

(c)

Fig. 4 SEM image of the nickel powder used on the MCF (a), SEM image of the metallic print lines on the MCF (b) and digital image of the MCF flat tape (c) 
Fig. 5 Printing of epoxy Nickel paste in the printing zone

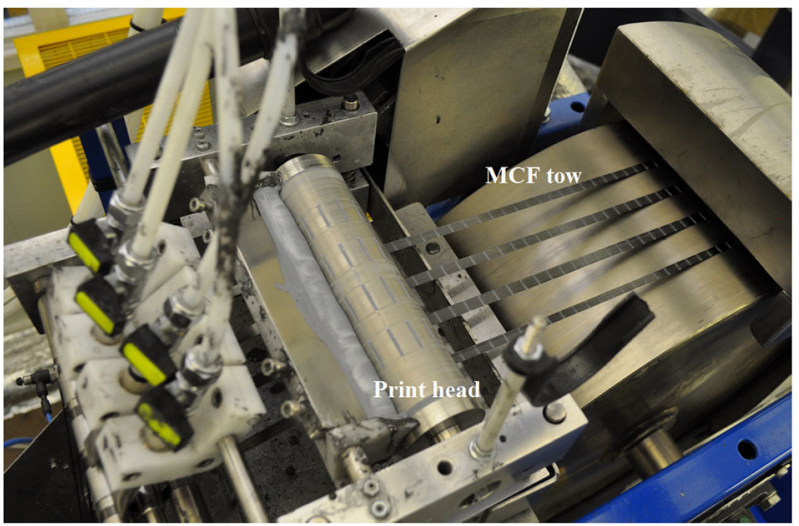

\section{Experimental}

The micro scale of the carbon fibres practically prevents the carbon being handled in the form of fibres. Therefore they need to be in the form of a bundle of fibres or tows. Specifically in the present research, Toray T700 60E carbon fibre tows having a width of 6-7 mm and a linear density of 800 Tex, consisting of 12,000 fibres was used. In processing these carbon tows to make them magnetisable, before the tows are chopped and placed on the mould surface, ferromagnetic material was incorporated into the carbon fibre tows in the form of intermittent transverse lines. For the present work dimensions of these lines were fixed at $1 \times 10.435 \mathrm{~mm}$ repeating at $10 \mathrm{~mm}$ intervals. The printing of these lines was carried out using a prototype MCF processing machine.

In practice an MCF tow is designed to be processed using a carbon fibre-specific chopper gun and may be chopped to a length of $<25 \mathrm{~mm}$ since no fibre placement difficulties are experienced due to not using any air evacuation systems [23, 27]. By placing the mould in a magnetic field and by controlling the direction of the magnetic force, it is possible to force the carbon to bend and assume the curvature of the mould surface itself. This allows the resin infusion to be carried out for consolidating the component being moulded, without the problem of fibre escape.

Therefore in using the chopped MCF tow, the important parameters are: the magnetic attraction required to pull and hold the chopped tows on to mould surfaces, the effect of the

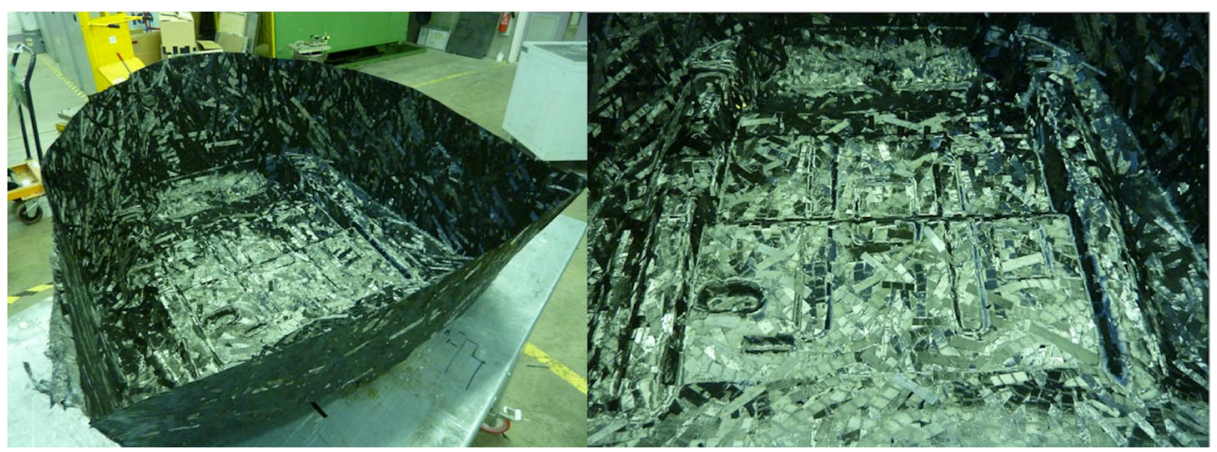

Fig. 6 Spare wheel well made using multifunctional carbon tow (Bentley Motors Ltd.) 


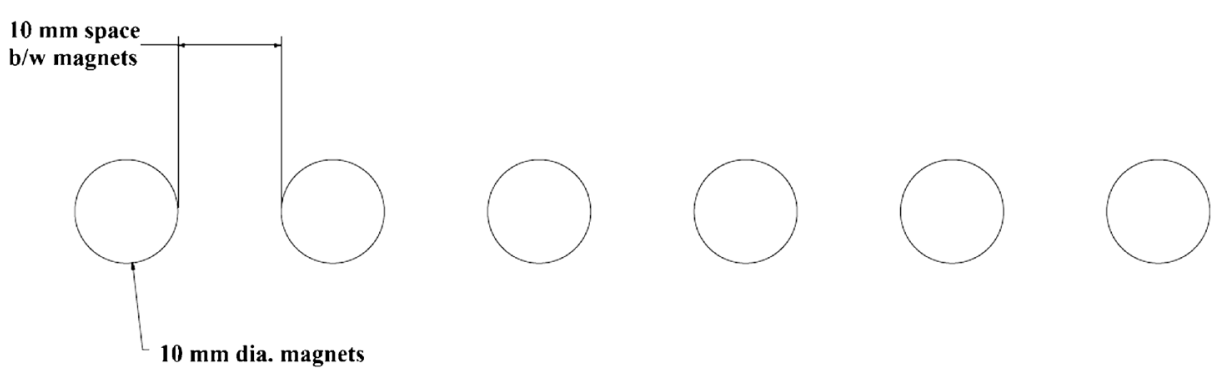

Fig. 7 Arrangement of the permanent magnets to create the magnetic pull force

orientation of the printed metallic lines in relation to the magnetic field, on the strength of the attraction force; and the strength of the magnetic field required to bend the chopped MCF on to the concave/convex mould surfaces. To observe the behaviour of the MCF tow under these conditions, magnetic pull force tests were conducted in a magnetic field, created by a set of 6 permanent disc magnets as shown in Fig. 7. The permanent magnets used were Neodymium-Iron-Boron disc magnets having a diameter of $10 \mathrm{~mm}$ and a thickness of $10 \mathrm{~mm}$.

In the test set up to observe the magnetic pull force on the MCF tow, illustrated in Fig. 8, these magnets were used, they were held in the cross bar of a $2.5 \mathrm{kN}$ Zwick/Roell tensile tester and the pull force was recorded using a load cell of $20 \mathrm{~N}$ at a cross head speed of $5 \mathrm{~mm} / \mathrm{min}$.

The test setup for observing the effect of relative position of the printed metallic lines to the permanent magnets was carried out by moving the tow sample in a lateral direction while the magnets were held stationary. To observe this magnetic pull force on a single printed line, the same test was conducted for a MCF tow having a single printed line.

In addition to these tests, to observe the bending properties of the MCF tow as a measure of its ability to assume the curvature of the mould cavity, a three point bending test was carried out according to the test standard BS ISO 5628:2012 shown in Fig. 9. In order to measure the bending properties of the metallised tow under the influence of a magnetic field, a test was conducted as shown in Fig. 10 using a set of disc magnets.

Fig. 8 Tensile tester setup for measuring the magnetic pull force

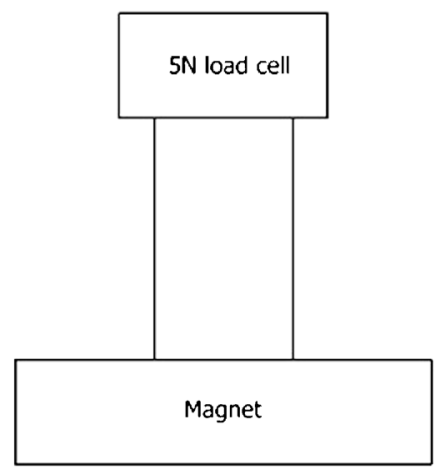


Fig. 9 Three point bending test

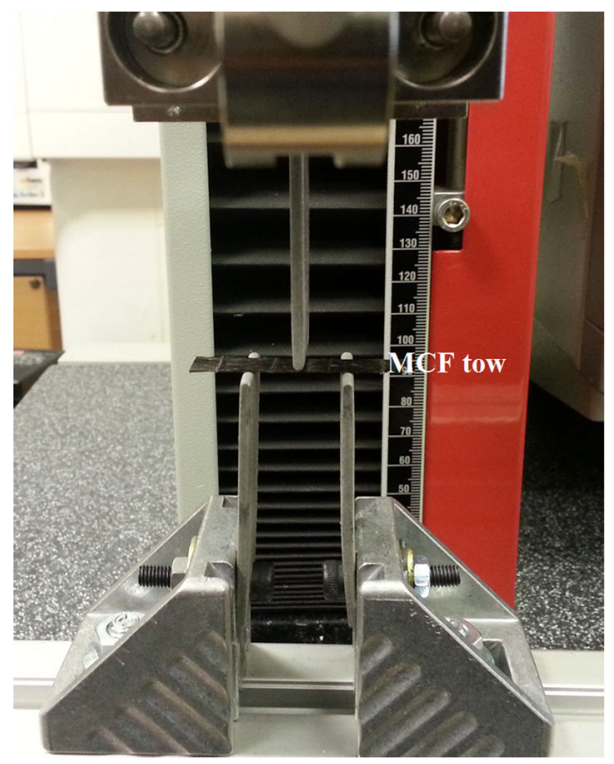

\section{Mathematical Model}

In order to determine the scope of the MCF tow for engineering applications, it is important to know, through scientific analysis, its magneto-mechanical performance as a preforming material in composites. To fulfil this aim, it is imperative that a process of mathematical modelling and scientific validation of the performance of MCF is carried out. Since the MCF consists of magnetically inert carbon fibres with periodically printed metallic lines on one side, to determine the performance of the MCF in a magnetic field an analysis of the effects of a magnetic field on the MCF tow need to be carried out. In this case, in the experimental environment, the magnetic field was created using an array of neodymium permanent disc magnets as illustrated in Fig. 10.

The pull force exerted on a single metallic line of the carbon tow in the experimental set up due to a single neodymium permanent disc magnet can be described using the theoretical relationships below [28]:

$$
F=A \frac{[B(x)]^{2}}{2 \mu_{0} \mu_{r}}
$$

Where

$F \quad$ magnetic pull force

$A$ area of the disc magnet

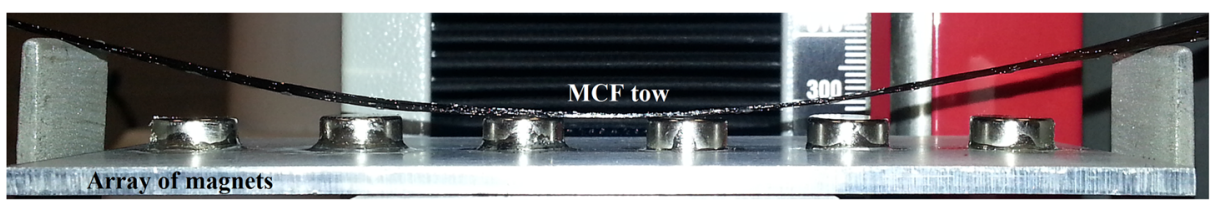

Fig. 10 MCF tow bending under the influence of the permanent magnetic field 
$B$ flux density on the MCF tow

$\mu_{0} \quad$ magnetic permeability of free space

$\mu_{r} \quad$ relative magnetic permeability

The flux density of the disc magnet at a distance ' $x$ ' from the magnet can be given by [29];

$$
B(x)=\frac{B_{r}}{2}\left[\frac{L+x}{\sqrt{R^{2}+(L+x)^{2}}}-\frac{x}{\sqrt{R^{2}+x^{2}}}\right]
$$

Where;

$B(x) \quad$ flux density at distance $\times$ from the magnet

$B_{r} \quad$ remanence of the magnetic material

$L \quad$ thickness of the magnet

$R \quad$ radius of the disc magnet

Combining the Eqs. (1) and (2)

$$
F=A \frac{\left[\frac{B_{r}}{2}\left[\frac{L+x}{\sqrt{R^{2}+(L+x)^{2}}}-\frac{x}{\sqrt{R^{2}+x^{2}}}\right]\right]^{2}}{2 \mu_{0} \mu_{r}}
$$

The relationship between the air gap height, from a magnet to the MCF tow, and the pull force, is defined by the Eq. 3. However in the present case, due to the multiple metallic lines and the array of permanent magnets in the setup, the magnetic pull force calculation is much more complex. Therefore the analysis in this case was carried out using the Ansys Workbench 14 suite of Finite Element Analysis software.

Since the carbon tow is magnetically inert, in the finite element analysis, only the effect of the magnetic field on the metallic lines was considered. For the finite element analysis, the material properties of nickel and neodymium permanent magnets were obtained from the ANSYS material properties. Due to the limitations on the computing power if the carbon fibres are modelled separately, the tow was defined as a homogenous tape having cross-sectional dimensions of 10.435 and $0.068 \mathrm{~mm} \mathrm{[30,}$ 31]. The effective dimensions of the printed nickel powder lines were kept at $10.435 \mathrm{~mm} \times 1 \mathrm{~mm} \times 0.4 \mu \mathrm{m}$.

The static magnetic and the static structural analysis environment was set up and analysed in the Ansys Workbench environment. The magnets used for the setup were neodymium permanent magnets and the lines printed on the carbon tows were modelled with the properties of nickel. For the static magnetic analysis the element type used was Solid117 3D magnetic solid. For the static structural analysis the element types used were Solid187 tetrahedral elements, Contact174 elements for contact analysis, Targe 170 for target faces, Surf154 for surface element modelling and Combin14 to represent the spring damper behaviour of the setup. Analysis settings also provides for large deflections in the setup. 
To simplify the analysis carried out, initially a static magnetic finite element analysis was carried out to observe the effect of the horizontal separation of a single Nickel powder printed line in relation to the magnet at a constant gap of $1 \mathrm{~mm}$ as seen in Fig. 11.

To observe the effect of an array of 6 magnets on a carbon tow printed with 7 lines of nickel powder mixed with epoxy, for the cases of relative horizontal and vertical position between the magnets and the printed lines as shown in Fig. 12, another two finite element analyses were carried out.

The bending rigidity of the MCF tow is an important parameter that will give an idea to the carbon tow's resistance to bending and thereby indications of the energy requirements of tow bending. In order to model the bending behaviour and the bending curvature of the MCF tow, initially a three point bending test was carried out as shown in Fig. 9. Thereafter the bending rigidity values derived through this test were used in the static structural bending models, aimed at gauging the bending curvatures of the MCF tow.

To carry this out, the magnetic attraction forces on the printed nickel lines were determined from the static magnetic analysis. Observing that the MCF tow tends to go through a sudden impulsive bending movement, if the magnets are brought too close, the tow-magnet separation was maintained at a minimum safe separation distance of $10.55 \mathrm{~mm}$. The results of this tow bending model were later validated using the maximum bending depth observed in the experimental setup.

\section{Results and Discussion}

The static magnetic and static structural tests were conducted with the aim of gauging the performance of the metallised carbon fibre tow when it is used together with the

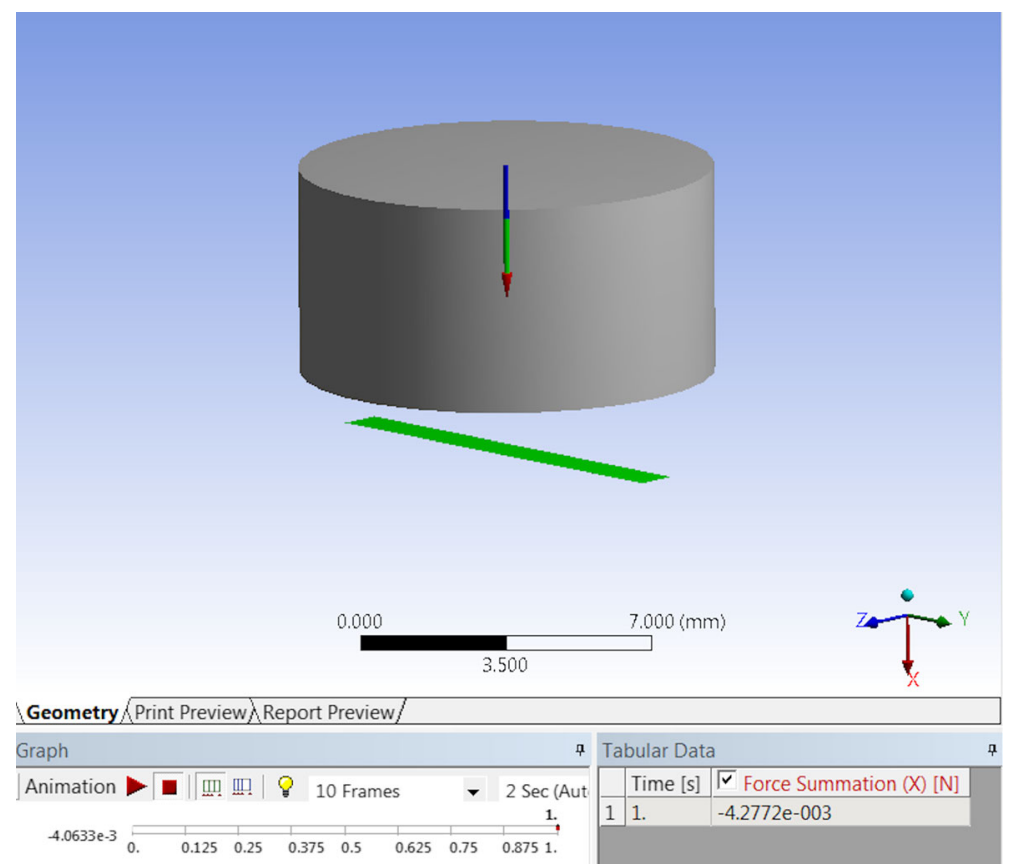

Fig. 11 Magnetic force on a single magnet due to a single nickel print line 


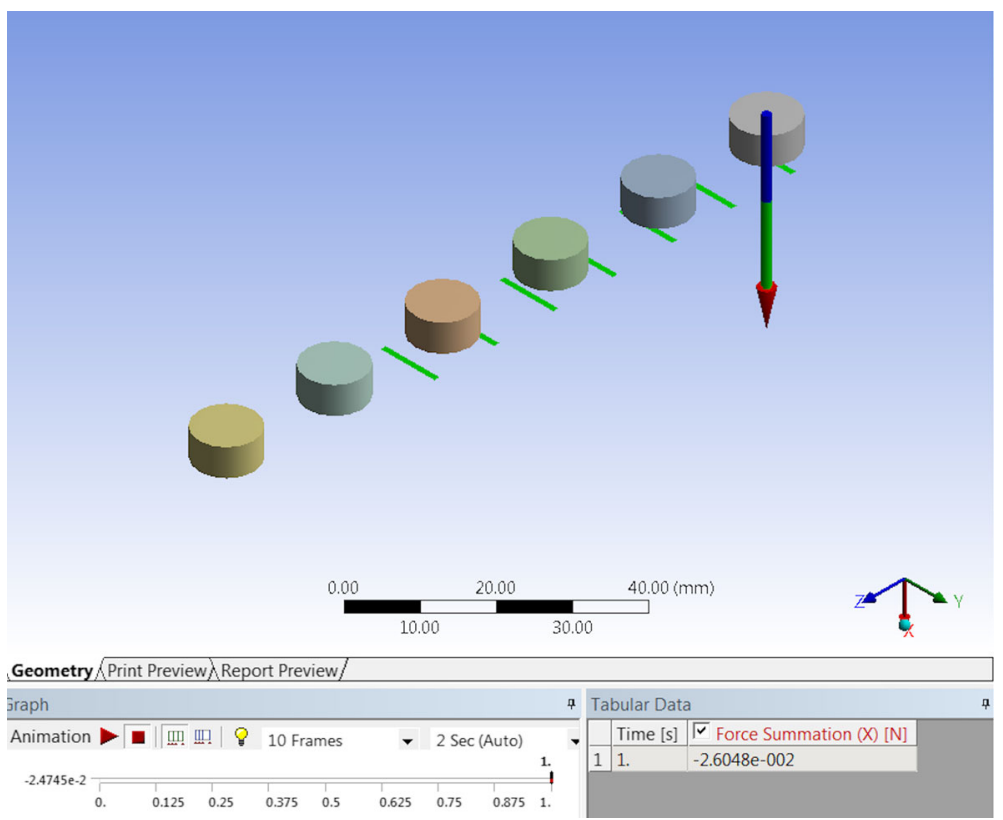

Fig. 12 Analysis of the effects of horizontal and vertical position of the magnets on the printed tow

magnetic tooling. The aim of the static magnetic tests was to see the effect of magnetic field strength on the MCF. By analysing the force experienced by the MCF depending on the separation between the MCF and the magnetic field, and the relative orientation between the magnetic field and the MCF, it would be possible to find the fluctuations of the force experienced by the MCF due to their positions. To this end experiments were conducted with both single magnet - single MCF line and multiple magnet - multiple line carbon tows. The results of these tests together with the results of the mathematical validation using Ansys Finite Element Analysis are given below.

\subsection{Single Line Single Magnet Test}

The experimental setup has shown that handling the MCF tow and the magnets at a distance closer than $2 \mathrm{~mm}$ is difficult. Therefore to conduct the experiments under an optimum magnetic force on the MCF tow, the separation was maintained at $2 \mathrm{~mm}$. The results were obtained with the horizontal displacement of the MCF tow from the centre of the tow by $1 \mathrm{~mm}$ steps.

The results in Fig. 13 show that the change in the intensity of the force experienced by the MCF due to a transverse tow displacement of half its width is negligible. This behaviour was qualitatively validated by the Ansys mathematical modelling. The finite element based mathematical model follows the empirical data closely in the centre of the magnet, where the flux lines are parallel and less closely towards the periphery of the magnet, where the flux lines are less predictable. The theoretical results are further explained by the graphical results presented in Fig. 14. It was observed that the highest intensity of the theoretical magnetic force is experienced at the ends of the metallic 


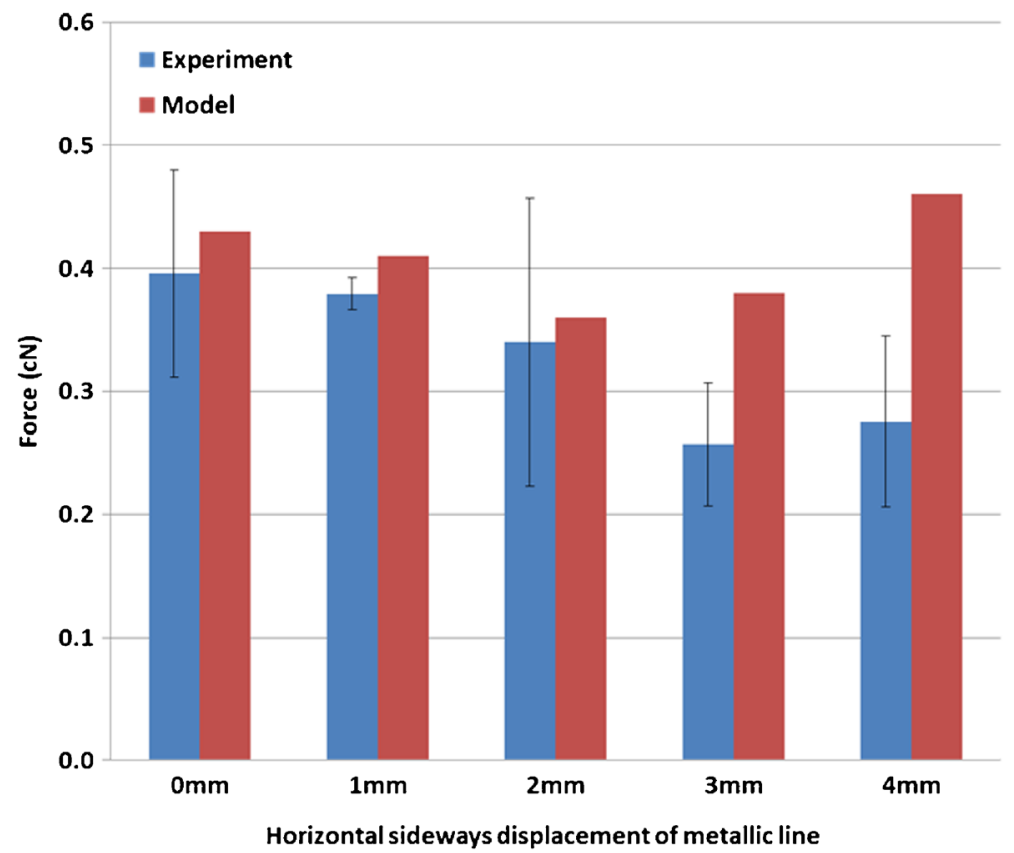

Fig. 13 Comparing the force experienced by the metallic line at a separation of $2 \mathrm{~mm}$, both theoretical and experimental, for horizontal sideways displacements of $1 \mathrm{~mm}$ from the centre

lines rather than the middle due to the nature of the finite element based mathematical models in Ansys.

Fig. 14 Total magnetic force $(\mathrm{N})$ experienced by the metallic line on the MCF tow due to a single cylindrical magnet

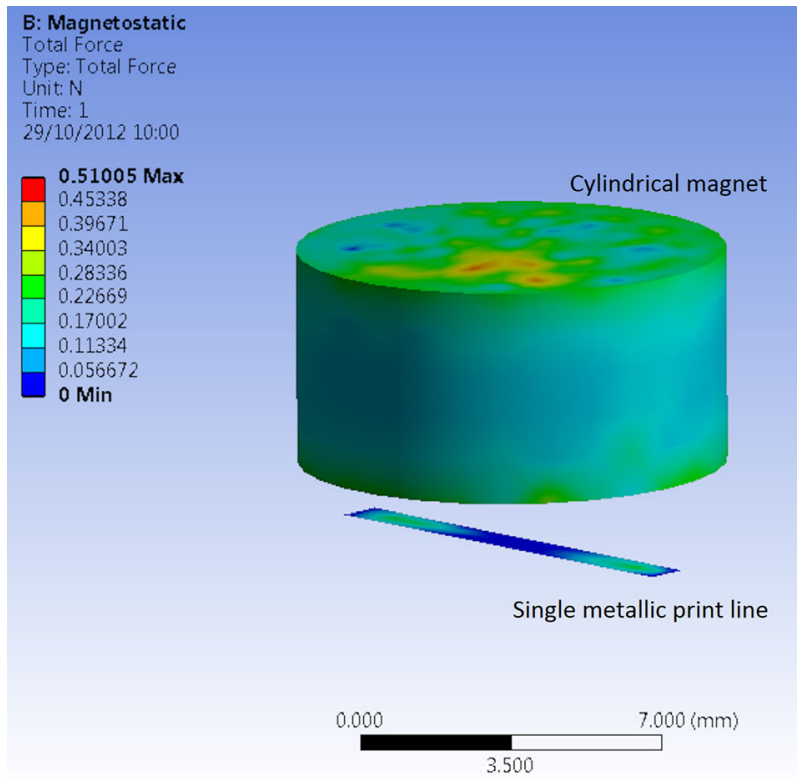




\subsection{Multi Line Multi Magnet Tests}

Although the single metallic line single magnet tests were conducted to observe the effect on a single metallic line, to observe the force field experienced by a length of chopped tow, multiline tow tests are essential. Therefore to provide these chopped tow lengths with a suitable magnetic field, an array of magnets was used.

\subsection{Horizontal Magnetic Field Shifting}

The theoretical analysis carried out to observe the effect of longitudinal displacement between the magnetic field and the MCF in the horizontal plane has shown in Fig. 15 that the change in force experienced by the MCF tow. This model verifies that it is possible to model a tool for the preforming process.

Therefore the current print line frequency of 1 line per $11 \mathrm{~mm}$ is sufficient to maintain a uniform force on all the printed metallic lines on the tow. The Ansys model created to represent this setup was validated by the experimental results shown in the bar chart given in Fig. 16.

\subsection{Vertical Magnetic Field Shifting}

As observed in Fig. 17 the force increases rapidly at small sepearation distances and decays as the sepearation increases. This information is very useful for determining the maximum thickness of the preform that can be made with the current metallic tape, for the magnetic force used in the experiments. As observed in the experiments, after $5 \mathrm{~mm}$ there is very small magnetic pull force to hold the tow in place. This information can be used for mould designing and as

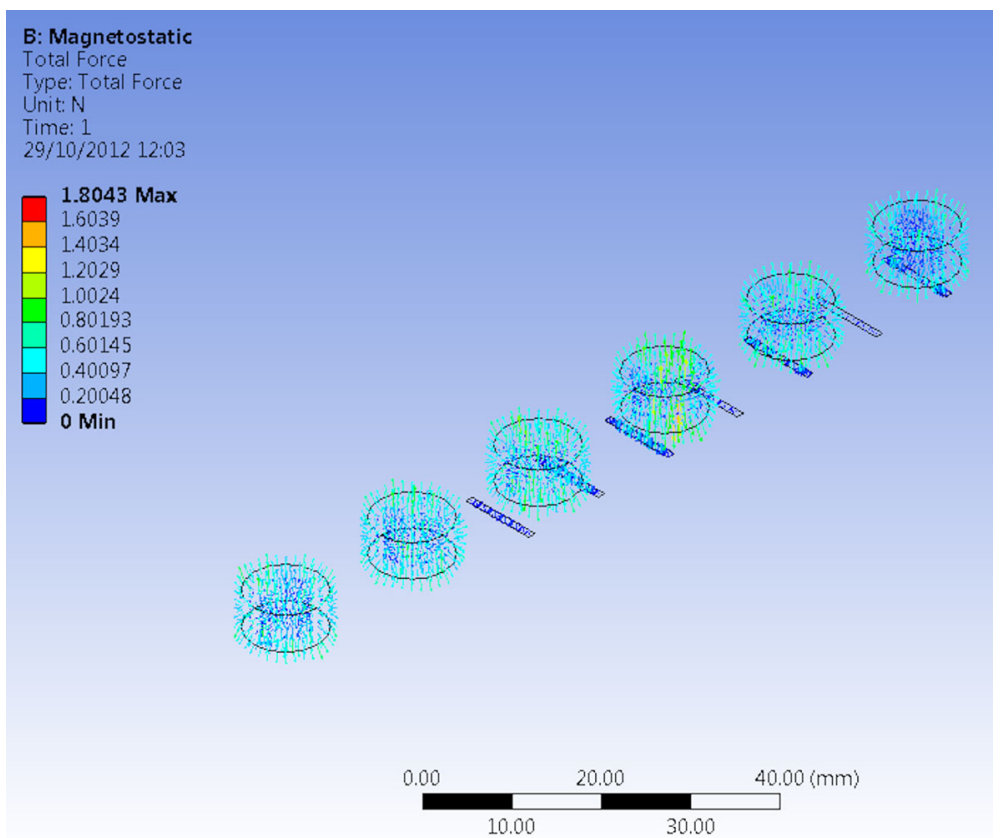

Fig. 15 Total force experienced by the metallic lines on the MCF tow due to the magnetic field 


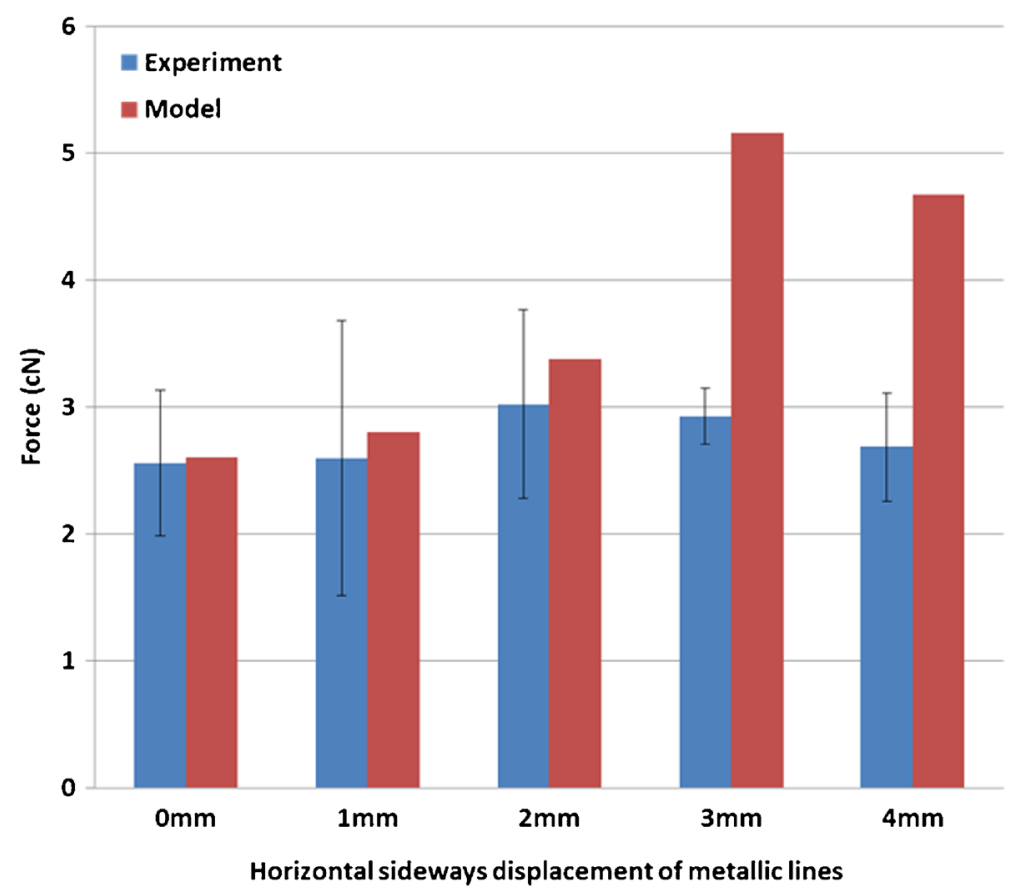

Fig. 16 Comparing the force experienced by the metallic lines at a separation of $2 \mathrm{~mm}$, both theoretical and experimental, for horizontal longitudinal displacements of $1 \mathrm{~mm}$ from the centre

directions for the determination of the magnetic force for a particular application, as certain applications need more pull force than others based on curvature/thickness of the mould.

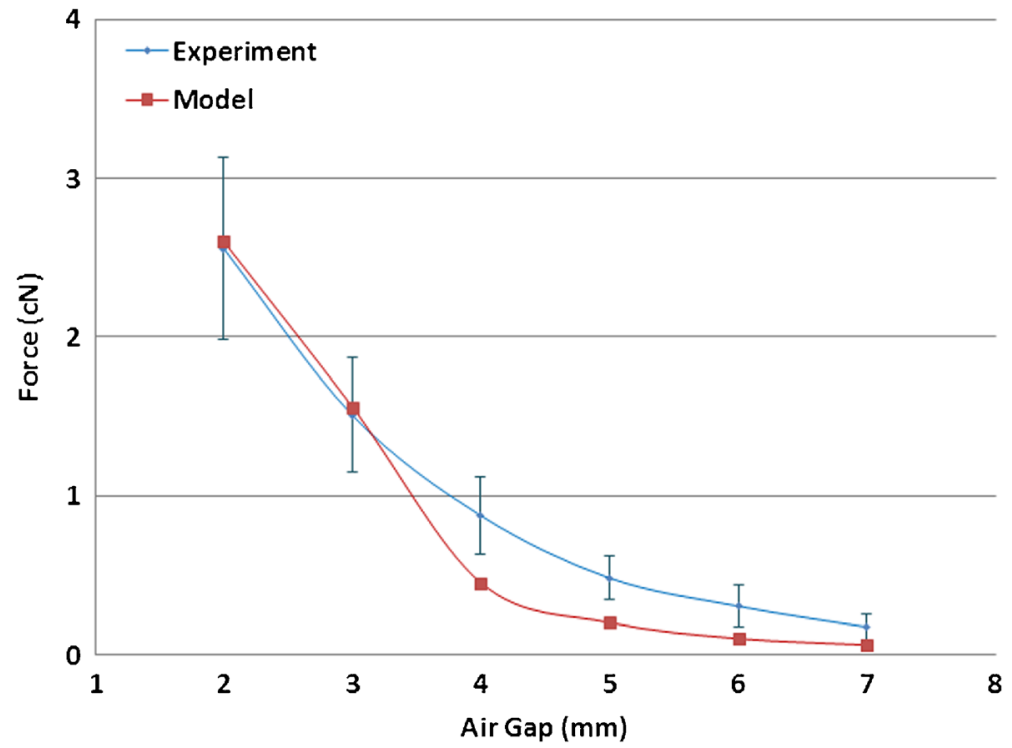

Fig. 17 The theoretical calculation of the magnetic force experienced by the MCF tow depending on the distance between the tow and the magnet array and its validation using experimental results 
Close validation can be observed between the Ansys model created to represent this setup and the experimental results.

\subsection{Three Point Bending Test}

The determination of the Young's modulus $(E)$ for modelling the MCF bending was carried out according to the standard BS ISO 5628:2012. All three specimens shown in Fig. 18 are single $12 \mathrm{k}$ carbon tow. For all specimen, the tow length is $50 \mathrm{~mm}$. Toray T700 60E tow had a width and thickness of 10.24 and $0.064 \mathrm{~mm}$ respectively. Average printed tow width and thickness for both the other specimen types in Fig. 18 are 10.63 and $0.072 \mathrm{~mm}$ respectively. According to this standard the relationship between the Young's modulus and the bending stiffness ( $\mathrm{Sb})$ is given by the following two equations;

$$
\begin{gathered}
S_{b}=\frac{E I}{b} \\
S_{b}=\left(\frac{F}{f}\right) \cdot\left(\frac{l^{3}}{3 b}\right)
\end{gathered}
$$

where

I Second moment of area,

$F$ force,

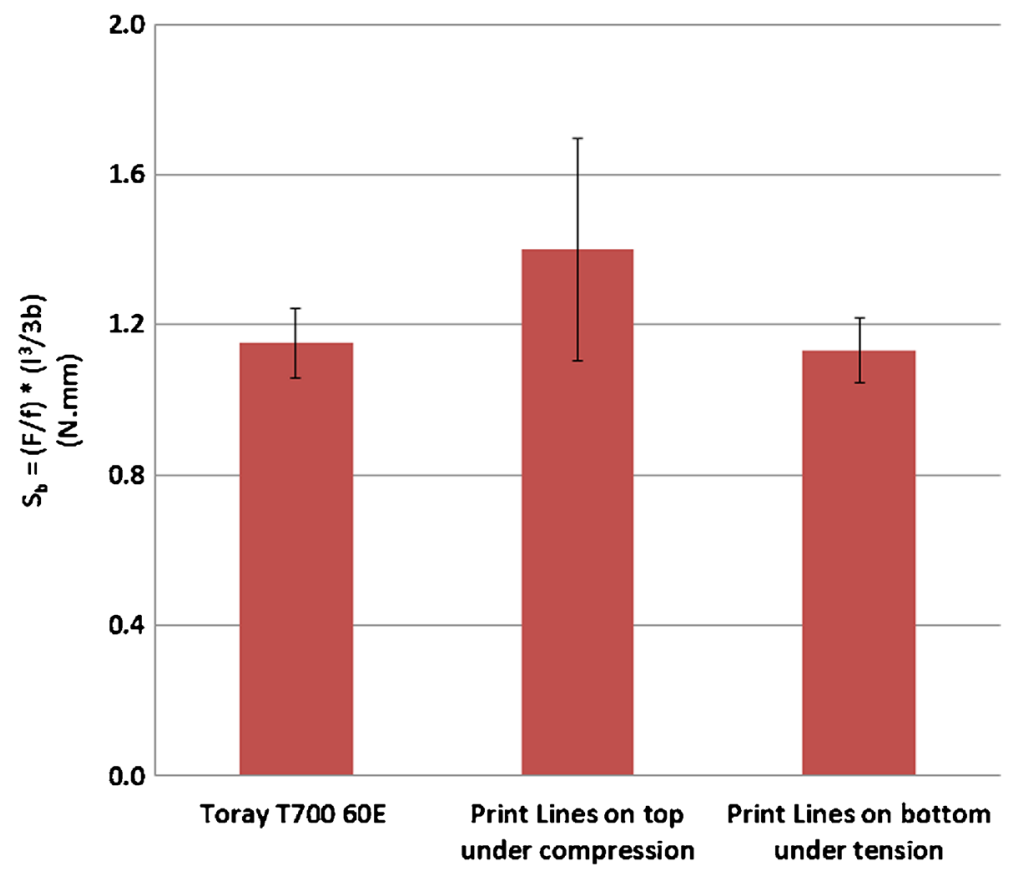

Fig. 18 Bending rigidity calculation for MCF tow according to the BS ISO 5628:2012. $F$ force, $f$ linear deflection, $l$ bending length, $\mathrm{b}$ specimen width 
$f$ linear deflection measured at the middle of the beam,

$l$ bending length,

$b$ specimen width

As Fig. 18 shows, the original bending stiffness of the carbon tow is increased due to the metallisation process. The bending stiffness is high under compression due to the resistance of the metallic print line. The quality of the print line also affects the stiffness data observed resulting in larger data scatter.

Also according to the figure, the bending direction, whether on the same side as the printed lines or in the opposite direction has little effect on the bending rigidity of the MCF tow. The Young's modulus value of $53 \mathrm{GPa}$ derived from the experimental results given in Fig. 18 and the Eqs. (4) and (5) from the standards were used for the MCF tow material definition in the Ansys analysis.

\subsection{Bending Deflection Under a Magnetic Field}

The mathematical modelling of MCF tow bending was carried out to observe its capacity to assume the curvature of a mould surface. The validation of the model (maximum theoretical deflection of $2.22 \mathrm{~mm}$ and the experimental value of $2.17 \mathrm{~mm}$ ) shows that the Ansys finite element model created for MCF tow bending, seen in Fig. 19 is closely validated by the experimental results. Also experimental observations show that beyond the maximum deflection of $2.17 \mathrm{~mm}$, the tow undergoes impulsive bending at very small bending radii due to the intense forces it experiences at close proximity to the magnet array.

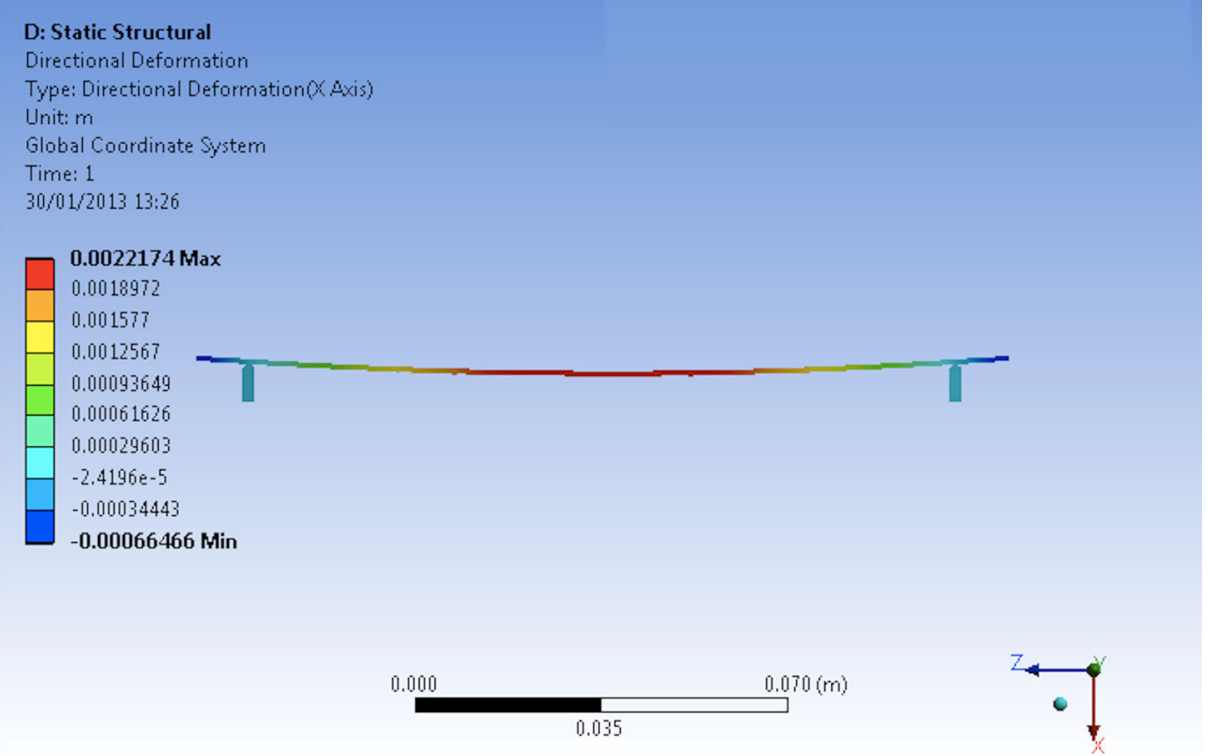

Fig. 19 Results of the 3 point bending model for the MCF tow having a length of $166 \mathrm{~mm}$, width $10.435 \mathrm{~mm}$ and a thickness of $0.068 \mathrm{~mm}$ 


\section{Conclusions}

Various iterations of machine designs that were studied have shown that the current design of the prototype MCF production machine is an efficent method for creating multifucntional carbon fibre flat tapes. Tests have shown that due to the narrow width of the tow and since the tow is designed to bend in the transverse direction in the mould, a pattern of transverse lines is the best way to create the MCF tow. The MCF manufacturing process has been designed in such a way as to define the alignment of the printed metallic lines with respect to the carbon fibre direction accurately. This way while achieving the optimum flexibility in the carbon tow, the required static magnetic performance is also met. Although the introduction of metallic lines necessitates the use of epoxy resins which unavoidably increases the rigidity of the tow, in this particular situation, tests have shown that the rigidity increased only by $10 \%$. Also it is recognised that much further work is needed to build on the success of this innovation to transfer this technology to manufacturing. Therefore, while maintaining the advantages already established by the DCFP process, the MCF takes the DCFP to an enhanced level of functionality and efficiency by allowing the use of magnetic tooling.

The static magnetic and static mechanical tests carried out on the output of the MCF manufacturing process developed at the University of Manchester, confirms the level of magnetic attraction, the ability of the magnetic field to handle the MCF tow, place it in a mould cavity and the ability to bend it to a radius as well. Further the non-elastic sudden impulsive bending the tow undergoes was seen to be reversible within limits. The observation of uniform empirical force distribution experienced by the tow for $1 \mathrm{~mm}$ displacements, as shown in Fig. 16, confirms that a uniform performance can be expected for the frequency and the dimensions of the metallic lines in the tow. However the determination of optimum line density in a tow and the optimum line dimensions and at the same time the optimum epoxy metal combination to be used in the case of MCF manufacturing would necessitate further experimentation to determine the pull force in each case.

The studies have shown that, to achieve optimum levels of the general quality parameters expected from the MCF tow, it is possible to systematically engineer the tow, so that for a particular magnetic field strength, it is possible to determine the optimum mould wall thickness, the print line width, print line frequency and the quantity of ferromagnetic powder in the lines. This information allows the optimum design of the print rollers, so that the general quality parameters are realised.

Acknowledgments This research is financially supported by Bentley Motors Ltd., United Kingdom.

Open Access This article is distributed under the terms of the Creative Commons Attribution 4.0 International License (http://creativecommons.org/licenses/by/4.0/), which permits unrestricted use, distribution, and reproduction in any medium, provided you give appropriate credit to the original author(s) and the source, provide a link to the Creative Commons license, and indicate if changes were made.

\section{References}

1. Frost \& Sullivan: automotive carbon fiber composites to grow at a staggering growth rate until 2017. http://www.frost.com/prod/servlet/press-release-print.pag?docid=263051000. Accessed 08 Feb 2013

2. The automotive industry and climate change Framework and dynamics of the $\mathrm{CO} 2$ (r)evolution. www.pwc. com/th/en/automotive/assets/co2.pdf. Accessed 08 Feb 2013 
3. Kyoto protocol. http://unfccc.int/kyoto_protocol/items/2830.php. Accessed 21 Feb 2013

4. Jambor, A., Beyer, M.: New cars - new materials. Mater. Des. 18(4-6), 203-9 (1997)

5. Suzuki, D., Takahashi, J., Kageyama, K. K. U., Ohsawa, I.: Purpose and target of the development of carbon fiber reinforced thermoplastics. SAMPE International Symposium \& Exhibition, Tokoyo, 27-30 Nov 2007

6. Ricardo weight savings study. http:/www.drivealuminum.org/research-resources/documents/2008-ricardoweight-savings-study (2008). Accessed 08 Feb 2013

7. Adam, H.: Carbon fibre in automotive applications. Mater. Des. 18(4-6), 349-55 (1997)

8. Dahl, J.S., Smith, G.L., Houston, D.Q.: The influence of fiber tow size on the performance of chopped carbon fiber reinforced composites. 37th ISTC-Seattle, WA, October 31- November 3, (2005)

9. Das, S.: Life cycle assessment of carbon fiber-reinforced polymer composites. Int. J. Life Cycle Assess. 16(3), 268-82 (2011)

10. Automotive world: time to lighten up. http://automotive.ihs.com/servlet/cats? pageContent= art\&serviceID=1675\& filterID=\&documentID=2514101\&typeID=0\&documentTypeId=64\&src=pc. Accessed 08 Feb 2013

11. Turner, T.A., Harper, L.T., Warrior, N.A., Rudd, C.D.: Low-cost carbon-fibre-based automotive body panel systems: a performance and manufacturing cost comparison. Proceedings of the Institution of Mechanical Engineers, Part D: Journal of Automobile Engineering. 222(1), 53-63 (2008)

12. Potluri, P., Atkinson, J.: Automated manufacture of composites: handling, measurement of properties and lay-up simulations. 34(6), 493-501 (2003)

13. Brandt, M.R., Reeve, S.R.: Directed Fibre Preform case studies. Proceedings of Composites 2001 convention and trade show, Tampa, FL USA, October, (2001)

14. Harper, L.T., Turner, T.A., Martin, J.R.B., Warrior, N.A.: Fiber alignment in directed carbon fiber preforms - a feasibility study. J. Compos. Mater. 43(1), 57-74 (2009)

15. Norris, R.E., Lomax, R.D., Xiong, F., Dahl, J.S., Blanchard, P.J.: Advanced high speed programmable preforming. info.ornl.gov/sites/publications/files/Pub22950.pdf. Accessed 10 March 2012

16. Rondeau, R., Reeve, S., Bond, G.: The effect of tows and filament groups on the properties of discontinuous fiber composites 44th International SAMPE Symposium and Exhibition, Long Beach, CA, USA, pp. 144960, May 23-27 1999

17. Chavka, N.G., Dahl, J.S.: P4: Glass fiber preforming technology for automotive applications. 44th International SAMPE Symposium, 23-27 May 1999

18. Cordell, T., Tolle, T.B., Rondeau, R.: The programmable powdered preform process for aerospace: affordable performance through composites. SAMPE International Symposium, May 2000

19. Reeve, S., Rondeau, R., Bond, G., Tervet, F.: Mechanical property translation in oriented, discontinuous carbon fiber composites. SAMPE International Symposium, May 2000

20. Feraboli, P., Cleveland, T., Ciccu, M., Stickler, P., DeOto, L.: Defect and damage analysis of advanced discontinuous carbon/epoxy composite materials. Compos. A: Appl. Sci. Manuf. 41(7), 888-901 (2010)

21. Reeve, S., Robinson, W.: Carbon fiber evaluation for directed fiber preforms. Proceedings of SAMPE 2001-Long Beach, CA, May, (2001)

22. Harper, L.T., Turner, T.A., Warrior, N.A., Dahl, J.S., Rudd, C.D.: Characterisation of random carbon fibre composites from a directed fibre preforming process: analysis of microstructural parameters. Compos. A: Appl. Sci. Manuf. 37(11), 2136-47 (2006)

23. Dodworth, A.: Bentley motors develops unique directional carbon fibre preforming process for chassis rails. 9th Automotive Composites Conference \& Exhibition- Michigan, September 15-16, (2009)

24. Harper, L.T., Turner, T.A., Martin, J.R.B., Warrior, N.A.: Fiber alignment in directed carbon fiber preforms - mechanical property prediction. J. Compos. Mater. 44(8), 931-51 (2010)

25. Harper, L.T., Turner, T.A., Warrior, N.A., Rudd, C.D.: Characterisation of random carbon fibre composites from a directed fibre preforming process: the effect of tow filamentisation. Compos. A: Appl. Sci. Manuf. 38(3), 755-70 (2007)

26. Harper, L.T., Dodworth, A., Luchoo, R., Warrior, N.A.: Automated spray deposition for net-shape carbon/ epoxy compression moulding. Proceedings of ICCM-17, Edinburgh, 27-31 July (2009)

27. Harper, L.T., Turner, T.A., Warrior, N., Rudd, C.D.: Characterisation of random carbon fibre composites from a directed fibre preforming process: the effect of fibre length. Compos. A: Appl. Sci. Manuf. 37(11), 1863-78 (2006)

28. IBS Magnet - formula to calculate the flux density. http://www.ibsmagnet.com/knowledge/flussdichte.php. Accessed 05 March 2012

29. Magnetic pull force. http://www.codecogs.com/reference/physics/magnetism/magnetic_pull_force.php. Accessed 08 March 2012

30. Potluri, P., Ramgulam, R., Chilo, M., Arshad, H.: Tow-scale mechanics for composite forming simulations. Key Eng. Mater. 504-506, 255-60 (2012)

31. Ramgulam, R.B., Potluri, P., Chilo, M.: Multiscale modelling: yarn deformation to draping simulation of woven preforms ICCM-17, Edinburgh, 27-31 July 2009 